\title{
COMPARATIVE STUDY OF THREE LINEAR SYSTEM SOLVER APPLIED TO FAST DECOUPLED LOAD FLOW METHOD FOR CONTINGENCY ANALYSIS
}

\author{
"Syafii, Hanalde Andre \\ Electrical Engineering Dept., Engineering Faculty, Universitas Andalas \\ *Corresponding author, e-mail : syafii@ft.unand.ac.id
}

\begin{abstract}
This paper presents the assessment of fast decoupled load flow computation using three linear system solver scheme. The full matrix version of the fast decoupled load flow based on XB methods used in this study. The numerical investigations are carried out on the small and large test systems. The execution time of small system such as IEEE 14, 30, and 57 are very fast, therefore the computation time can not be compared for these cases. Another cases IEEE 118, 300 and TNB 664 produced significant execution speedup. The superLU factorization sparse matrix solver has best performance and speedup of load flow solution as well as in contigency analysis. The invers full matrix solver can solved only for IEEE 118 bus test system in 3.715 second and for another cases take too long time. However for superLU factorization linear solver can solved all of test system in 7.832 second for a largest of test system. Therefore the superLU factorization linear solver can be a viable alternative applied in contingency analysis.
\end{abstract}

Keywords : Fast Decoupled Load Flow, Contigency Analysis, and Linear System Solver

Copyright $\odot 2017$ JNTE. All rights reserved

\section{INTRODUCTION}

The contingency analysis is one of the basic tools utilized in control centers. This tool is used to help the operation engineers to take appropriate and fast decisions to respond for events such as component outages. The execution time of the power flow adopted in the contingency analysis is very critical. The classical approach to speed up the solution process is to use approximate power system model. This approach has been in use for many years and goes under various names such as linear sensitivity method using DC load-flow or distribution factor methods [1]. The former methods have limitations attributed to only branch MW flows are calculated and these are only within $5 \%$ accuracy, hence, the MVAR flows or bus voltage magnitudes are still unknowns [1].

The knowledge of the MVAR flows and bus voltage magnitudes after a contingency outage requires executing complete $\mathrm{AC}$ power flow. This presents a great deal of difficulty when thousands of cases must be computed, then the total time to test for all of outages will be too long. Due to the problem, other methods are used to run complete load flow on some critical cases.
These techniques go under the name of concentric relaxation [2], bounding method for AC contingency screening $[3,4]$ and artificial intelligent based method [5]. The problem of these techniques is requires more layer and consumed longer computing time.

This paper describes the implementation of three linear solvers in load flow algorithm base on fast decoupled method. The computation time of complete fast decoupled load flow in contingency analysis will be investigated.

\section{LOAD FLOW ANAL YSIS}

Load flow analysis is used in planning and designing the future expansion of power systems, as well as in system operation [6]. Load flow also provides steady state condition for other analysis such as stability studies, short-circuit and outage security assessment. In general, the load flow involves the solution of a set of nonlinear equations given by:

$$
\mathbf{F}(\mathbf{x})=\mathbf{J} . \Delta \mathbf{x}
$$

Where;

$F(x)$ is a set of nonlinear equations with the same number of variables. 
$\mathrm{J}$ matrix is the Jacobian matrix, which is a set of the differential equation of $\mathrm{F}(\mathrm{x})$ with respect to each of the variables $\mathrm{x}$.

$\Delta \mathrm{x}$ is a vector correction of variables $\mathrm{x}$ in the $\mathrm{F}(\mathrm{x})$ function.

Based on (1), the load flow solution is written in terms of power equations as:

$$
\left[\begin{array}{c}
\Delta \mathbf{P} \\
\Delta \mathbf{Q}
\end{array}\right]=\left[\begin{array}{cc}
\mathbf{H} & \mathbf{N} \\
\mathbf{J} & \mathbf{L}
\end{array}\right] \cdot\left[\begin{array}{c}
\Delta \boldsymbol{\theta} \\
\Delta \mathbf{V}
\end{array}\right]
$$

\subsection{Fast Decoupled Method}

The fast-decoupled load flow (FDLF) developed by Stott and Alsac [7] is the most popular one because of its simplicity and computational efficiency. The FDLF formulation is developed by neglecting the $\mathrm{J} 2$ and $\mathrm{J} 4$ submatrices of the Jacobian matrix in (2). This approximation can be applied since the real power changes $(\Delta \mathrm{P})$ are less sensitive to the change in voltage magnitude $(\Delta \mathrm{V})$ and more sensitive to the change of phase angle $(\Delta \theta)$. On the other hand, $\Delta \mathrm{Q}$ is more sensitive to the change of $\Delta \mathrm{V}$. Due to this coupling characteristics, (2) can be simplified into:

$$
\left[\begin{array}{c}
\Delta \mathbf{P} \\
\Delta \mathbf{Q}
\end{array}\right]=\left[\begin{array}{cc}
H & \cdot \\
\cdot & \mathrm{L}
\end{array}\right] \cdot\left[\begin{array}{c}
\Delta \theta \\
\Delta \mathrm{V}
\end{array}\right]
$$

This lead to decoupled equations:

$$
\begin{aligned}
& \Delta P=H . \Delta \theta \\
& \Delta Q=L . \Delta V
\end{aligned}
$$

The above equations show that the matrix equation has been separated into two decoupled equations that solve $\mathrm{P}-\theta$ and $\mathrm{Q}-\mathrm{V}$ problem separately and iteratively.

These equations can be simplified by setting the voltage $\mathrm{V}=1.0$ unit and the $\mathrm{H}$ and $\mathrm{L}$ element are divided with the bus voltage. The resulting FD load flow equations after further simplification are then given:

$$
\begin{aligned}
& \Delta P / V=B^{\prime} \Delta \theta \\
& \Delta Q / V=B^{\prime \prime} \Delta V
\end{aligned}
$$

The matrix $\mathbf{B}^{\prime}$ and $\mathbf{B}^{\prime \prime}$ are the negative of the imaginary part of the nodal admittance matrix $\mathbf{Y}$, without the column and row corresponding to the slack node. The FD algorithm involves the following steps:

1. Calculate $\mathbf{B}^{\prime}$ and $\mathbf{B}^{\prime \prime}$ and its inversion or factorize matrices

2. Calculate the initial mismatches $\Delta \mathbf{P} / \mathbf{V}$

3. Solve equation (6) for $\Delta \boldsymbol{\theta}$

4. Update the angle $\theta$ and use it to calculate $\Delta \mathbf{Q} / \mathbf{V}$

5. Solve equation (7) and update $\Delta \mathbf{V}$

6. Return to step 2 and repeat the procedure until all mismatches are within specified tolerances.

Although, the FD method uses the approximation of Jacobian matrix, the mismatch equations are calculated using exact equations. This makes the solution process as accurate as in the original Newton-Raphson method. Due to the simple equations involved in FD method, the FD implementation has more advantageous over NR in terms of execution time, memory requirements, and at the same it can be implemented as parallel algorithm [8].

The analytical analysis of load flow analysis requires a linear solver to solve sparse linear equation in the form of $\mathrm{A} . \mathrm{x}=\mathrm{b}$. In this paper, a public domain library, SuperLU [9], is used as the solver for load flow analysis. It is used since it uses many latest techniques, such as graph reduction technique in matrix factorization. Furthermore, very unsymmetrical matrices can also be solved using this library. The library comes with four packages containing real and complex solvers, in both single and double precision versions

\subsection{Fast Decoupled Load flow for Contingency Analysis}

The Contingency analysis which is run in an operations and control center must be executed very quickly. There are three basic ways to accomplish this [1], that are: 1). With approximate but very fast algorithms, 2). Select only the importance cases for detailed analysis, $3)$. Use the computer system made up of multiple processors to gain speed.

The first technique using sparse matrix for complete load flow have adopted in this research. The simples and accurate contingency analysis consists of running AC power flow analysis for each generator, transmission line and transformer outage as shown in Figure 1. The 
fast linear solver makes a program run faster and more efficient. Inside the contingency loop, B' and B" development and factorization repeated until all component outage finish tested. The load flow iteration scheme also repeated for all components contingency, but in this part not more consumed time calculation, because of variable updated for every calculation step in order to increase convergence achievement.

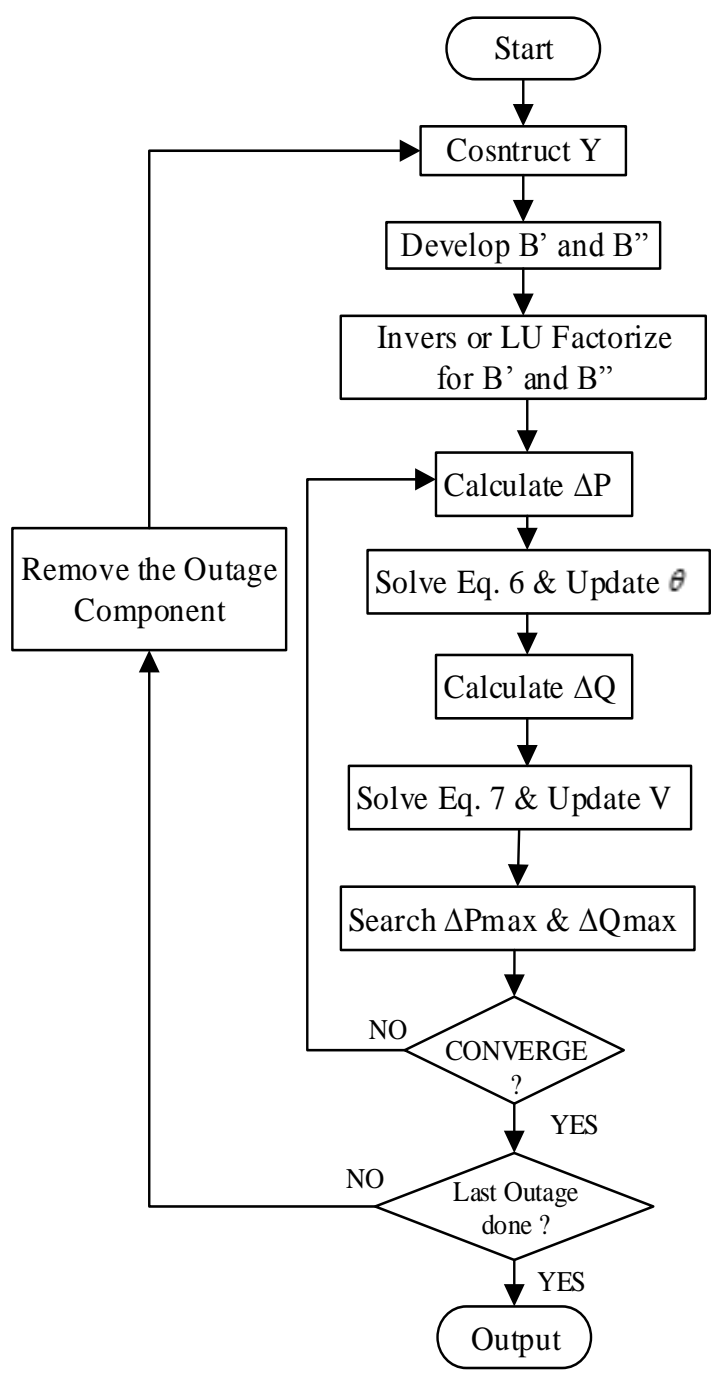

Figure 1. Fast Decoupled Algorithm with Contingency Analysis

In fast decoupled load flow computation, it is common to deal with very large matrices where only a few elements are not zero. The B' and B" Factorization matrix has leads to a large consumed execution time in the processing even though one times factorized. In such cases, memory consumption can be reduced and performance increased by using a specialized representation storing only the nonzero coefficients. Such a matrix is called a sparse matrix.

\section{DESCRIPTION OF THE TEST SYSTEM}

The IEEE data and Tenaga Nationa Berhad (TNB) already choosen for system test. The IEEE 30, 57, 118, 300 bus and TNB 664 bus data have been used to test full and spare matrix based linear solver of fast decoupled load flow method. The contingency analysis running full and spare matrix based linear solver of Fast Decoupled load flow for each possible transmission line outage. The IEEE 118 and 300 bus with comprises of 179 and 411 transmission line respectively. Therefore, load flow analysis resolved 179 times for IEEE 118 bus and 411 times for IEEE 300 bus data. For TNB 664 bus, the contingency only for 275 and $400 \mathrm{kV}$ transmission line or 304 times load flow analysis resolved.

The IEEE 30, 57, 118 and 300 bus are provided in ref [10]. In contingency analysis IEEE 118, 300 bus and TNB 664 bus already used to present large scale power system data. Then system tested under Intel Pentium dualcore CPU $1.8 \mathrm{GHz}$.

\section{RESULT AND DISCUSSION}

The single fast decoupled load flow computation result shown in Table 1 and Tabel 2. For small system IEEE 14, 30, and 57 the execution time is very fast, Therefore the time computation cannot be compared for these cases. The another cases IEEE 118, 300 and TNB 664 produced significant execution speedup. The fast decoupled load flow results using invers of full and sparse matrix solver are given in Table 1 and the fast decoupled load flow results using invers full matrix and superLU factorization solver are given in Table 2. The more efficient and fastest solver is superlLU factorization specially for large scale system as shown in Table 2 . Since the application of superLU factorization has better performance, this linear solver is very attractive to be applied in contingency analysis. 
Table 1. Fast decoupled load flow results using invers full and sparse matrix solver

\begin{tabular}{|c|l|c|c|c|c|c|c|}
\hline \multirow{2}{*}{ No. } & \multirow{2}{*}{ Test Cases } & \multicolumn{5}{|c|}{ Algorithm } \\
\cline { 3 - 8 } & & Time & Iteration & Losses & Time & Iteration & Losses \\
\cline { 3 - 8 } & & 0.0117 & 4 & 13.5302 & 0.0078 & 4 & 13.5302 \\
\hline 1. & IEEE 14 Bus & 0.0117 & 4 & 17.5793 & 0.0313 & 4 & 17.5795 \\
\hline 2. & IEEE 30 Bus & 0.0313 & 5 & 28.3834 & 0.0391 & 5 & 28.3839 \\
\hline 3. & IEEE 57 Bus & 0.1289 & 5 & 179.4460 & 0.0899 & 5 & 179.4458 \\
\hline 4. & IEEE 118 Bus & 1.7693 & 7 & 417.3891 & 0.2695 & 8 & 417.3877 \\
\hline 5. & IEEE 300 Bus & 18.378 & 8 & 167.9541 & 0.9375 & 8 & 168.1888 \\
\hline 6. & TNB 664 Bus & & & & & & 5 \\
\hline
\end{tabular}

Table 2. Fast decoupled load flow results using invers full matrix and superLU factorization solver

\begin{tabular}{|c|l|c|c|c|c|c|c|}
\hline \multirow{2}{*}{ No. } & \multirow{2}{*}{ Test Cases } & \multicolumn{6}{|c|}{ Algorithm } \\
\cline { 3 - 8 } & & \multicolumn{3}{|c|}{ Invers Full Matrix } & \multicolumn{3}{c|}{ SuperLU Factorization } \\
\cline { 3 - 8 } & & Time & Iteration & Losses & Time & Iteration & Losses \\
\hline 1. & IEEE 14 Bus & 0.0117 & 4 & 13.5302 & 0.0117 & 4 & 13.5302 \\
\hline 2. & IEEE 30 Bus & 0.0117 & 4 & 17.5793 & 0.0117 & 4 & 17.5795 \\
\hline 3. & IEEE 57 Bus & 0.0313 & 5 & 28.3834 & 0.0195 & 5 & 28.3839 \\
\hline 4. & IEEE 118 Bus & 0.1289 & 5 & 179.4460 & 0.0195 & 5 & 179.4457 \\
\hline 5. & IEEE 300 Bus & 1.7693 & 7 & 417.3891 & 0.0508 & 8 & 417.3877 \\
\hline 6. & TNB 664 Bus & 18.378 & 8 & 167.9541 & 0.0977 & 8 & 168.1888 \\
\hline
\end{tabular}

Table 3. Contigency analysis computation time results using invers full matrix and superLU factorization solver based.

\begin{tabular}{|l|c|c|c|c|c|c|}
\hline \multicolumn{1}{|c|}{ Cases } & \multicolumn{2}{c|}{ IEEE 118 } & \multicolumn{2}{c|}{$\begin{array}{c}\text { IEEE 300 } \\
\text { (1711 line outage) }\end{array}$} & \multicolumn{2}{c|}{$\begin{array}{c}\text { TNB 664 } \\
\text { (304 line outage) }\end{array}$} \\
\hline Linear Solver & $\begin{array}{c}\text { Invers Full } \\
\text { Matrix }\end{array}$ & $\begin{array}{c}\text { SuperLU } \\
\text { Factorization }\end{array}$ & $\begin{array}{c}\text { Invers Full } \\
\text { Matrix }\end{array}$ & $\begin{array}{c}\text { SuperLU } \\
\text { Factorization }\end{array}$ & $\begin{array}{c}\text { Invers Full } \\
\text { Matrix }\end{array}$ & $\begin{array}{c}\text { SuperLU } \\
\text { Factorization }\end{array}$ \\
\hline $\begin{array}{l}\text { Develop B'\&B” \& } \\
\text { Linear Eq.System }\end{array}$ & 0.403 & 0.061 & na & 0.609 & na & 1.533 \\
\hline Iteration & 2.591 & 0.392 & na & 5.228 & na & 6.145 \\
\hline Total Time & 3.715 & 0.562 & na & 6.428 & na & 7.832 \\
\hline
\end{tabular}

na = no answer; more then 1 hour.

In contingency analysis full fast decoupled are repeated for every loop of contingency. Therefore B' and B" development and it's factorize executed many time in the program. The contingency analysis running Fast Decoupled load flow for each possible transmission line outage. The IEEE 118 and 300 bus with comprises of 179 and 411 transmission line respectively. Therefore load flow analysis resolved 179 times for IEEE 118 bus and 411 times for IEEE 300 bus data. For TNB 664 bus, the contingency only for $275 \mathrm{kV}$ and $400 \mathrm{kV}$ transmission line or 304 line outage.
The Contigency analysis computation time results using invers full matrix and superLU factorization solver based given in Table 3 . The invers full matrix solver can solved only for IEEE 118 bus test system in 3.715 second and for another cases take too long time. However for superLU factorization linear solver can solved all of test system in 7.832 second for a largest of test system. Therefore the superLU factorization linear solver can be a viable alternative applied in contingency analysis or other repetitive power system computation. 


\section{CONCLUSION}

The paper have presents the assessment of three linear system solver scheme applied to the fast decoupled load flow. The full matrix version of the fast decoupled load flow based on XB methods used in this study. The numerical investigations are carried out on the small and large test systems. The execution time of small system such as IEEE 14, 30, and 57 are very fast, therefore the time computation can not compared for these cases. Another cases IEEE 118, 300 and TNB 664 produced significant execution speedup. The superLU factorization sparse matrix solver have best performance and speedup of load flow solution as well as in contigency analysis. The invers full matrix solver can solved only for IEEE 118 bus test system in 3.715 second and for another cases take too long time. However for superLU factorization linear solver can solved all of test system in 7.832 second for a largest of test system. Therefore the superLU factorization linear solver can be a viable alternative in contingency analysis.

\section{ACKNOWLEDGEMENTS}

The author gratefully acknowledge the assistance rendered by the Faculty of Engineering, Universitas Andalas for partially funding this research.

\section{REFERENCES}

[1] A.J Wood and B F Wollenberg, " Power Generation Operation and Control, John Wiley \& Sons, InC, 1996.

[2] J. Zaborsky, K.W. Whank, K. Prasad, “ Fast Contingency Evaluation using Concentrix Relaxation", IEEE Transaction PAS, Vol PAS-99, January/February 1980, pp 28-36.

[3] Brandwajn, V., "Efficient Bounding Method for Linear Contingency Ananlysis", IEEE on Power System, Vol 3 No.1 February 1988, pp 38-43.

[4] Syafii and Nurul Rahmawati, "Analisis Kontingensi Sistem Tenaga Listrik dengan Metode Bounding", Jurnal Rekayasa Elektrika, Universitas Syiah Kuala, Vo.10 No.2, Okt 2012
[5] I. S. Saeh and A. Khairuddin, "Static security assessment using artificial neural network," in Proc. IEEE Int. Conf. Power and Energy,Dec.2008, pp. 1172-1177.

[6] D. Issicaba and J. Coelho, "Rotational Load Flow Method for Radial Distribution Systems", International Journal of Electrical and Computer Engineering (IJECE), ISSN: 2088-8708, Vol. 6, No. 3, June 2016, pp. 1344 1352

[7] Stott, B. and Alsaç, O. "Fast Decoupled Load Flow", IEEE Transaction PAS, vol 93, pp 859-869, 1974.

[8] Syafii, Khalid M Nor, Ali Khazejadeh and Mamdouh Abdel-Akher "Application of Parallel Processing in Fast Decoupled Load Flow Analysis", International Conference EEIES 2009, December 7-8, 2009 at Parkroyal Penang, Malaysia

[9] J. W. Demmel, S. C. Eisenstat, J. R. Gilbert, X. S. Li, and J. W. H. Liu, "A supernodal approach to sparse partial pivoting", SIAM Journal on Matrix Analysis and Applications, vol. 20, no. 3, pp 720 -755, 1999.

[10] Power Systems Test Case Archive: http://www.ee.washington.edu/research/ps tca

\section{About the Authors}

Syafii received B.Sc degree in electrical engineering from University of North Sumatera, and M.T. degree in electrical engineering from Bandung Istitute of Technology, Indonesia, in 2002 and Ph.D degree from Universiti Teknologi Malaysia in 2011. He is currently a senior lecture in Dept. of Electrical Engineering, Universitas Andalas, Indonesia. His research interests are new and renewable energy, smart grid and power system computation.

Hanalde Andre, received B.Sc degree in electrical engineering from Universitas Andalas, in 2011 and M.T. degree in electrical engineering under double degree program between Universitas Andalas and Bandung Istitute of Technology, Indonesia in 2013. He is currently a lecture in Dept. of Electrical Engineering, Universitas Andalas, Indonesia.. 